\title{
Impurities Effect on Carbonate Reactive Crystallization for the Wastewater
}

\author{
Y. Shimizu and I. Hirasawa \\ Department of Applied Chemistry, School of Advanced Science and Engineering, Waseda University, 3-4-1 Okubo, \\ Shinjuku-ku, Tokyo 169-8555, Japan \\ Correspondence should be addressed to Y. Shimizu; yoshimethod@gmail.com
}

Received 14 December 2012; Accepted 17 December 2012

Academic Editors: F. Lefebvre, J. Subrt, and C. Xu

Copyright ( 2013 Y. Shimizu and I. Hirasawa. This is an open access article distributed under the Creative Commons Attribution License, which permits unrestricted use, distribution, and reproduction in any medium, provided the original work is properly cited.

\begin{abstract}
Reactive crystallization designed to separate nickel or copper ion from effluents has been advanced for applying to actual industrial wastewater containing impurities. In the primary reaction of this method, metal sulfate solution reacts with sodium carbonate solution in a semibatch crystallizer. In the present study, during the process of nickel or copper ions incorporation, inhibitory effect on seed growth of impurities, like cobalt, manganese, zinc, and borate and phosphate ions, was investigated. Through the 8hour reactive crystallization, obtained particles' characters and metals removal efficient were examined. Considering analyses data on metal component ratio in produced crystals, metal ions initial uptake rate was found to be different by the kind of seeds and impurities. And the centrifugation was performed against obtained crystals aimed for examining target metal purity improvement. The results indicated that copper components can incorporate and remove other metal ions easily. In addition, when the anions are used as impurities, depending on the kind of anions, the effect of damaging the surface of seeds or producing many fine particles has been confirmed.
\end{abstract}

\section{Introduction}

Metal ions in the wastewater have often been treated by coagulation and precipitation methods [1]. In this treatment, however, a large amount of sludge is produced and metal substances are disposed without recycling. Meanwhile, wastewater treatment technologies based on environmental crystallization have an advantage in collecting metal ions as solid crystals. For example, studies about metal ion separation and precipitation methods with the use of a fluidized bed reactor have been preceded [2-4]. Some metal ions were shown to be taken efficiently on seeds such as quartz-sands at an optimum $\mathrm{pH}$. And in our recent report [5], in a study using semibatch crystallizer, we suggested that metal ions were recovered on seeds regularly and continuously at only particular seed inputs. Actually, in the industrial effluent, many kinds of metal ions are contained typically. Metal ions uptake mechanism somewhat relates to the adsorption or coprecipitation process. Some have found the order of metal ions adsorption strength to some base seeds [6-8], and others have examined the $\mathrm{pH}$ range at which metal ions can be selectively separated efficiently with the use of difference of solubility products in the process of co-precipitation $[9,10]$.

Thereby, in this paper, on the basis of nickel or copper ions crystallization in the regulated solution, seeds growth inhibition mechanisms by the presence of one type of other ions were examined. In the primary reaction, a metal sulfate solution was reacted with a sodium carbonate solution. And as impurities, cobalt, manganese, and zinc divalent ions or anions, borate and phosphate ions were mixed in the stock solution and reacted. In the process of target and impurity ions uptake in seeds, resultant particles' surface roughness, sizes and metals weight ratio were examined. Along with observing crystals growth via 8-hour reactive crystallization, metals initial uptake rate was calculated. In addition, the time variation with metal ions concentration in the effluent filtrate was analyzed. Thereafter, the centrifugation was performed against resultant metal suspending solution, and purity improvement by the difference of specific gravity was confirmed. 


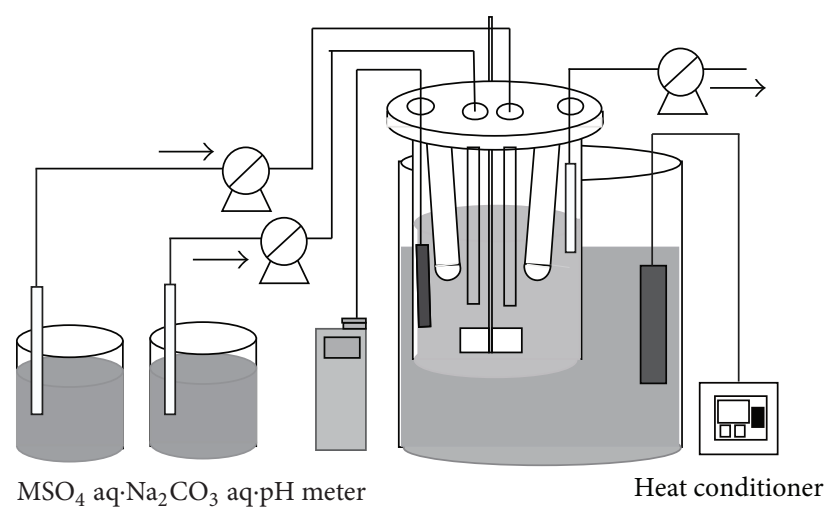

(a)

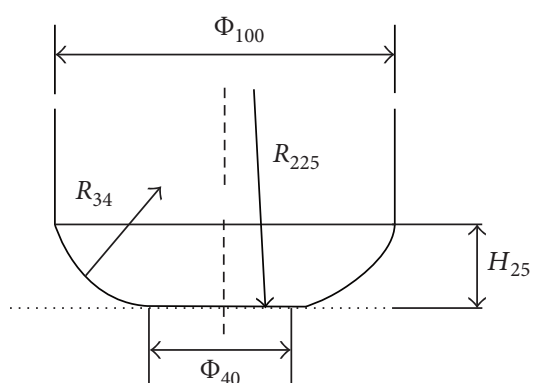

(b)

FIGURE 1: Schematic illustration of the semibatch crystallizer ( $M$ is a divalent metal ions blend) (a) and the geometry of a custom-ordered tank (b), (H: height, $R$ : radius of sphere, and $\Phi$ : inner diameter of the circular cylinder, $(\mathrm{mm}))$.
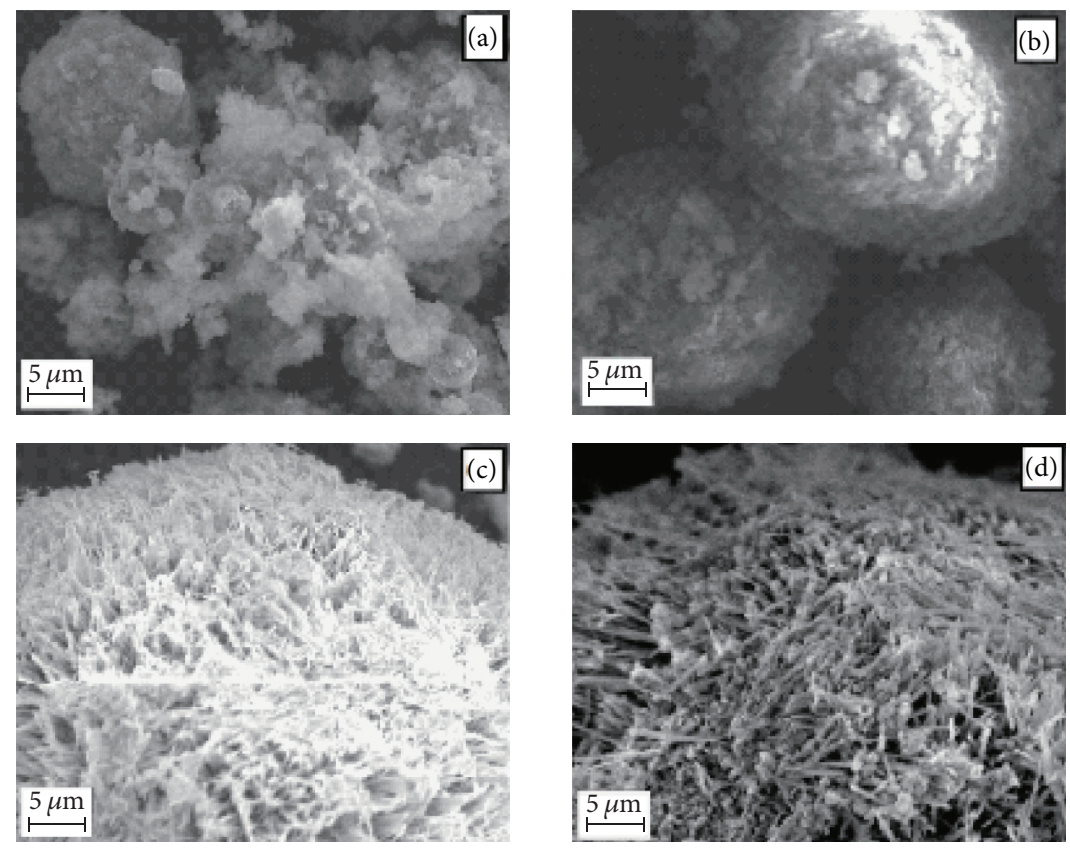

FIGURE 2: Grown seeds SEM images observed after the reactive crystallization ((a) nickel/manganese, (b) nickel/zinc, (c) copper/cobalt and (d) copper/zinc ions removal experiment).

\section{Material and Methods}

2.1. Laboratory Equipment and Reactive Conditions. Semibatch reactive crystallization was operated in a columnshaped tank which was equipped with a heat conditioner and an agitator, as shown in Figure 1. The detail geometry of custom-ordered tank has been introduced in our previous paper, [5]. Spherical seeds: metal carbonate basic (Wako Pure Chemical Industries, Ltd., Osaka, Japan), are placed preliminarily in the reaction tank. As the source of target metal ions, $\mathrm{NiSO}_{4} \cdot 6 \mathrm{H}_{2} \mathrm{O}$ or $\mathrm{CuSO}_{4} \cdot 5 \mathrm{H}_{2} \mathrm{O}$ (Wako) solution was used and impurities were provided in the form of $\mathrm{CoSO}_{4} \cdot 7 \mathrm{H}_{2} \mathrm{O}, \mathrm{MnSO}_{4} \cdot 6 \mathrm{H}_{2} \mathrm{O}, \mathrm{ZnSO}_{4} \cdot 7 \mathrm{H}_{2} \mathrm{O}$, boric acid, or phosphoric acid (Wako) solution. The solution containing
$1000 \mathrm{mg} \mathrm{L}^{-1}$ target and one type of impurity ions was coordinated by deionized water. And sodium carbonate (Wako) solution was prepared in the same way as the other stock solution. At this time, carbonate ions concentration in the sodium carbonate solution was regulated at sufficient amount to keep the optimum reaction molar ratio during the reaction. Each solution was provided at the feeding rate of $0.010 \mathrm{~L} \mathrm{~min}^{-1}$ into the tank on a double-jet basis. At the same time, the supernatant effluent was drained to keep the solution volume in the tank constant: $1.3 \mathrm{~L}$. The solution temperature in the tank was maintained at $303 \pm 0.1 \mathrm{~K}$. At any experiment, the agitation rate was set so as to be constant the boundary line between crystals and supernatant. To investigate the mechanism of incorporating metal ions 


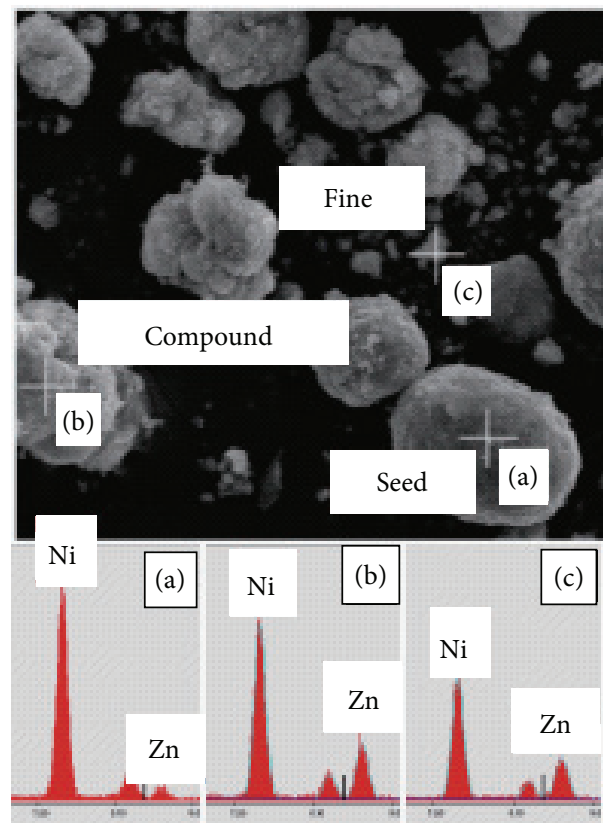

FIGURE 3: Grown seeds SEM images observed after the reactive crystallization and the number of composing metal ions (nickel/zinc ion removal experiment. Vertical scales: the number of metal ions (-) and horizontal scales: ions energy (keV), (a) seed, (b) compound, and (c) fine particle).

onto seeds, 8-hour reactive crystallization was conducted. Seed input was $40 \pm 0.1 \mathrm{~g}$ in nickel ion removal experiment and $20 \pm 0.1 \mathrm{~g}$ in copper ion removal experiment because this is the optimum amount for crystallization, keeping the form is sphere and restricting the fine particles production. And the initial $\mathrm{pH}$ of stock solution was controlled at each optimum value for the crystallization: at $9.7 \pm 0.1$ for nickel ion removal experiments and at $13.0 \pm 0.1$ for copper ion removal experiments.

2.2. Measurement Items. Supernatant and mixed suspension in the tank was sampled at regular interval during the reaction. Obtained crystals character and metals distribution on surface of produced crystals were observed by scanning electronic microscope (SEM: VE-8800, KEYENCE, Osaka and $\mathrm{H} 8100 \mathrm{~A} / 200 \mathrm{kV}$, Hitachi, Ltd., Tokyo) installed with energy dispersive X-ray spectrometry (EDX). And also in the tests of using metal ions as impurities, the content of specific metal in produced crystals was measured by X-ray fluorescence analysis (XRF: ZSX Primus II, RIGAKU, Tokyo). From these data, metal ion initial uptake rate per seed surface area $R_{T 0}$ and $R_{I 0}$ were determined. Furthermore, suspended solutions obtained after 8-hour crystallizations were centrifuged at the rate of $10000 \mathrm{rpm}$ and for 30 minutes by centrifugal machine (MX-301, Tomy, Tokyo) and examined for their purity improvement. Metal ions concentration in the supernatant filtrate was analyzed by inducted coupled plasma optical emission spectrometry (ICP: IRIS-Intrepid, Thermo Fisher Scientific K. K., Yokohama). The experiments described in this paper were repeated at least three times, with similar results.

\section{Results and Discussion}

3.1. Obtained Crystals Properties. After performing 8-hour reactive crystallization against metal ions stock solutions, obtained crystals were observed by SEM, as shown in Figure 2. In the presence of manganese ions for impurities, they were reacted with carbonate ions selectively, and spherical manganese carbonate small grains have been clearly formed (Figure 2(a)). Meanwhile, in the presence of zinc or cobalt ions for impurities, they crystallized just like target ions and incorporated into seeds uniformly (Figures 2(b) and 2(d)). Even when impurities exist in the reaction, seed growth was observed as well as when no impurities exist, granular fine particles were produced in the nickel ions incorporation experiment and needle-like crystals grew from the center of seeds at the copper ions incorporation experiment. And among obtained crystals, three types of components: seeds, compounds, and fine particles were found (Figure 3). From the EDX analytical data of grown nickel carbonate basic particles, target and impurity ions were found to be incorporated in fine particles and compounds at similar ratio. And almost all particles were mainly composed of target ions. This indicates that target metal ions are incorporated in seed particles easily.

3.2. Metal Ions Uptake Rate into Seed Crystals. Time variation of target metal components existence mole ratio constructing produced crystals is illustrated in Figure 4. In these metal ions uptake experiments, target metal mole balance equation (1) was considered and metal ions uptake rate: $r_{T}$ for target ions and $r_{I}$ for impurity ions were approximated. And in this equation, $m_{0}$ is metal mole in seeds and $\mathrm{M}_{T}$ represents target 


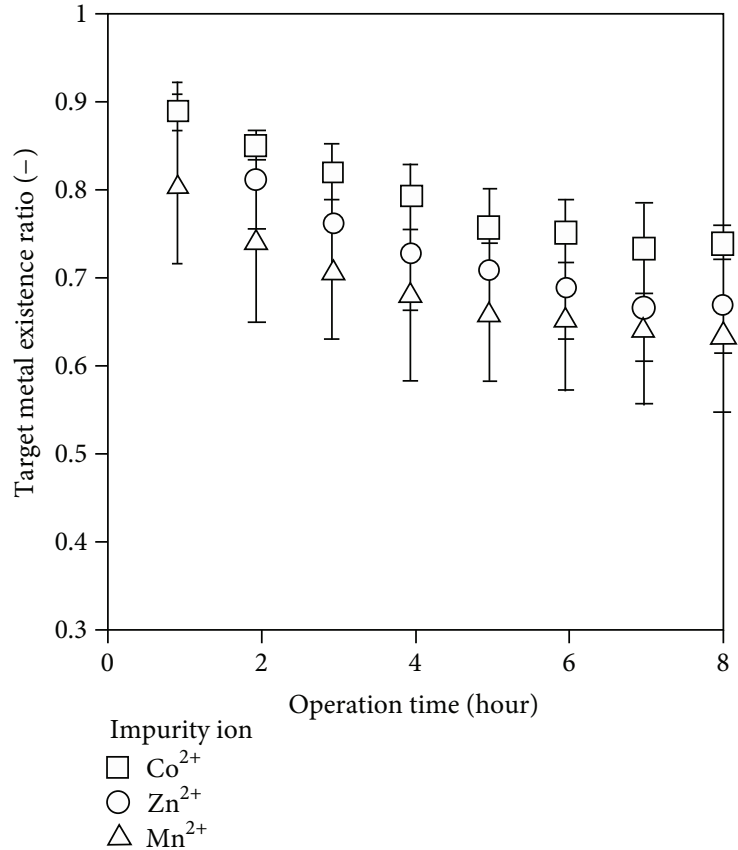

(a)

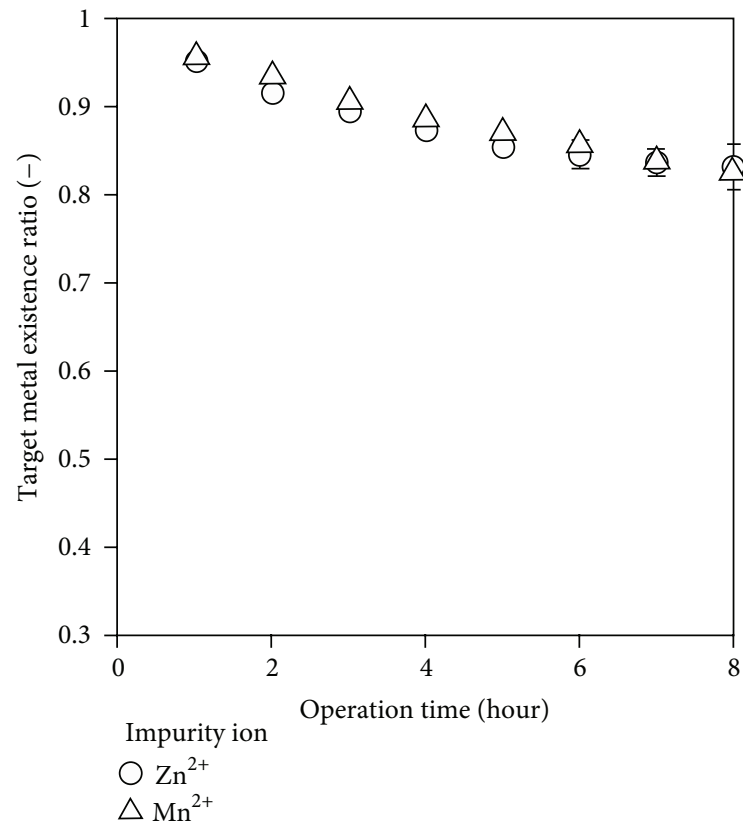

(b)

FIGURE 4: Time variation of the existence of mole ratio of target metal components in sampled crystals ((a) the experiment based on copper carbonate basics, (b) the experiment based on nickel carbonate basics).

TABLE 1: Estimated values of metal ions initial uptake rate per unit surface area.

\begin{tabular}{lcccc}
\hline Seeds & \multicolumn{2}{c}{ Copper carbonate basic } & \multicolumn{2}{c}{ Nickel carbonate basic } \\
impurity & $R_{T 0}\left(10^{-8} \mathrm{~mol} \mathrm{~m}^{-2} \mathrm{~s}^{-1}\right)$ & $R_{I 0}\left(10^{-8} \mathrm{~mol} \mathrm{~m}^{-2} \mathrm{~s}^{-1}\right)$ & $R_{T 0}\left(10^{-9} \mathrm{~mol} \mathrm{~m}^{-2} \mathrm{~s}^{-1}\right)$ & $R_{I 0}\left(10^{-9} \mathrm{~mol} \mathrm{~m}^{-2} \mathrm{~s}^{-1}\right)$ \\
\hline $\mathrm{Co}^{2+}$ & 3.343 & 1.840 & Not available & Not available \\
$\mathrm{Zn}^{2+}$ & 2.432 & 2.128 & 2.120 & 0.6056 \\
$\mathrm{Mn}^{2+}$ & 6.605 & 4.674 & 3.293 & 0.8706 \\
\hline
\end{tabular}

metal mole ratio in seeds. And from these data, target and impurity ions initial uptake rate per unit surface area: $R_{T 0}$ and $R_{I 0}$ were calculated using total input seeds surface area $A_{0}$ (2) and (3). Seeds surface area was estimated from 300 seed particles measured sizes (4). Estimated incorporation rate values were summarized in Table 1 . The reason why initial values were applied is that crystals surface area has been changing intricately during the reaction, and the $R$ value can also be changing:

$$
\begin{gathered}
\mathrm{M}_{T}=\frac{\left(m_{0}+r_{T} \cdot t\right)}{\left(m_{0}+r_{T} \cdot t+r_{\mathrm{I}} \cdot t\right)}, \\
R_{T 0}=\frac{r_{T}}{A_{0}}, \\
R_{I 0}=\frac{r_{I}}{A_{0}}, \\
A_{0}=A_{\text {unit }} \cdot N .
\end{gathered}
$$

Initial uptake rate to nickel carbonate basic is not fast and $R_{I 0}$ value has been about one fourth that of $R_{T 0}$. Copper and impurity ions both were incorporated readily in copper carbonate basic, and the proportion of $R_{I 0}$ and $R_{T 0}$ was from half to one. The reason of this ease of uptake to copper is estimated to relate to high metal ion adsorption force. Another reason seems that copper carbonate basics form needle-like products in this initial reaction and they can enlarge the uptake site.

3.3. Specific Weight Separation by Centrifugation. Suspension was centrifuged and solid contents were divided by the difference of specific weight. After the centrifugation, target metal mole ratio in condensate was analyzed by XRF (Figure 5). Target metal mole ratio was improved by from 10 to $20 \%$ in experiment based on copper carbonate basic. Meanwhile, this value did not change in experiment based on nickel carbonate basic. Part that cannot be separated even by the gravity separation seemed to be incorporated inside of crystals on the reaction process. Purity improvement in copper experiment is likely related to easily removable nature of copper. And 


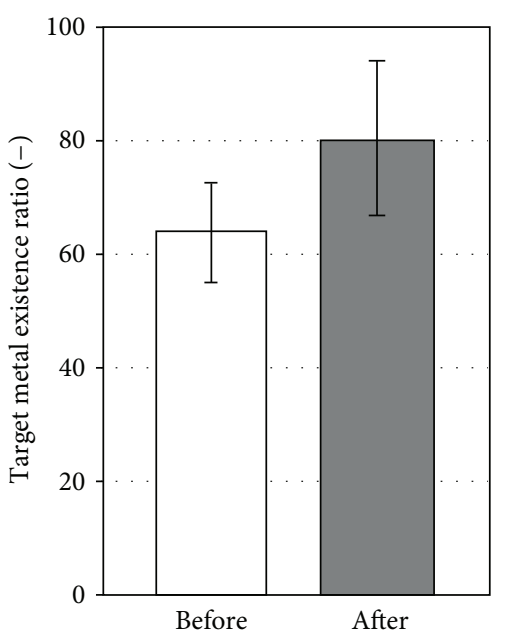

(a)

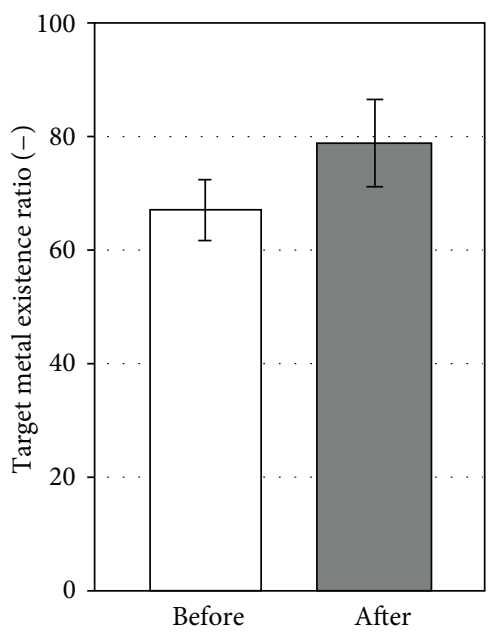

(c)

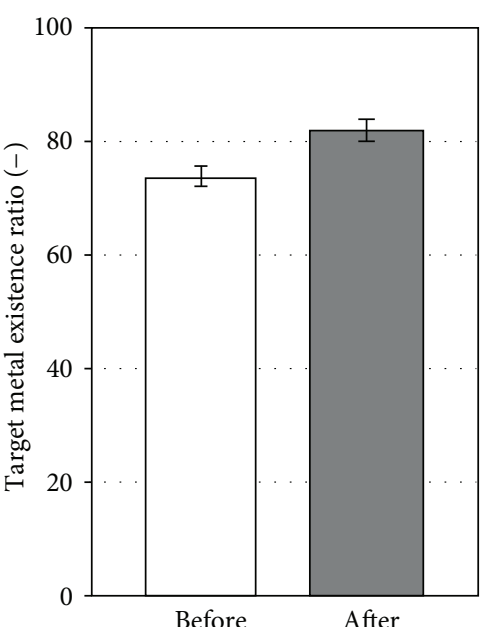

(b)

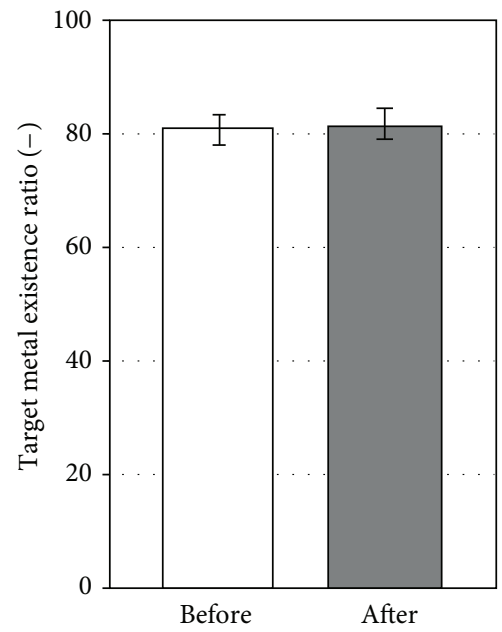

(d)

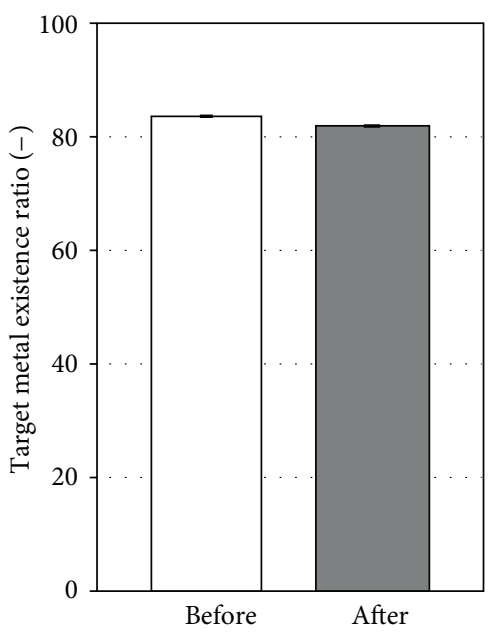

(e)

Figure 5: Target metals mole ratio before and after the centrifugation ((a) copper/cobalt, (b) copper/manganese, (c) copper/zinc, (d) nickel/zinc, and (e) nickel/manganese ions removal experiment). 
Borate ions uptake experiment

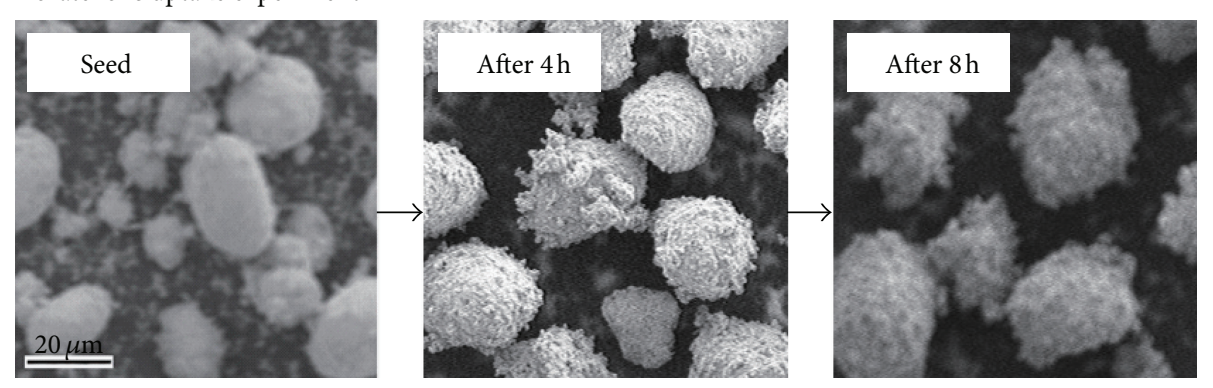

Phosphate ions uptake experiment

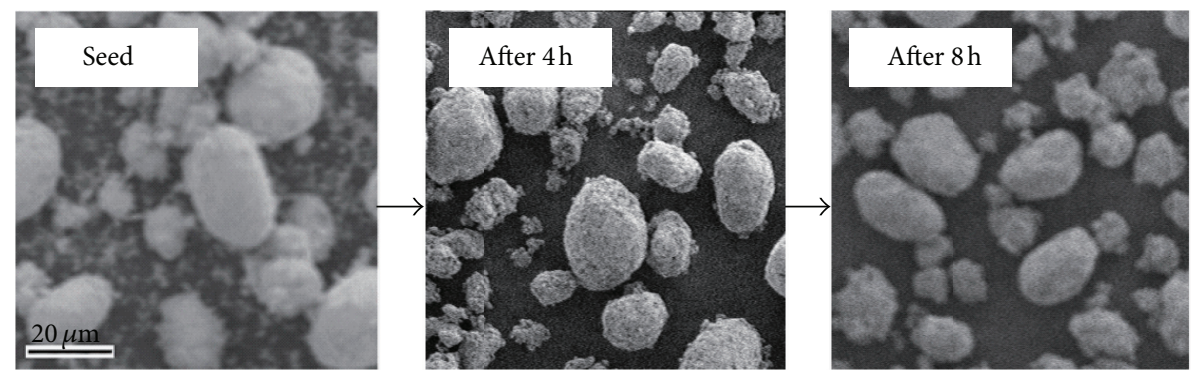

FIGURE 6: Grown seeds crystal SEM images in providing $0.1 \mathrm{M}$ anion impurities.

purity seems to have definite ceiling in around $80 \%$ in this condition.

3.4. Metal Ion Concentration in Supernatant. Target and impurity ions concentration in the effluent supernatant filtrate were both almost below $10 \mathrm{mg} \mathrm{L}^{-1}$ on an average during the reaction. This indicates that target metal components and impurities in stock solution were both removed about $99 \%$. And this means that metal ions separation operation by the reactive crystallization is efficient to remove metal ions. Also in the next stage, the separation and the purification of obtained crystals will come to be notable questions.

3.5. Anion Impurities Effect on Seed Crystals. In providing boric acid and phosphoric acid as anion impurities during the 8-hour reaction, time variation of produced crystals appearance, average size, and C. V. value are described in Figures 6 and 7. It has been found that the seeds growth mechanism was different by the kind of anions. When borate ions were provided as impurities, seeds surface has gotten rough, but the particles average size has been enlarged gradually because the particulates production was restricted. Meanwhile, in providing phosphate ions as impurities, with the increase of the anions concentration, more fine particles have been produced and the particles average size has been smaller. Particularly in this case, it has been found that even the existence of low-concentration anions enormously affected the seed growth. And these results indicate that this kind of anions gives the various effects to this reaction. As for the C. V. value, the regular change by the anions addition has not been found out.

\section{Conclusions}

The results indicated that heterogeneous metal ions had inhibitory effect against target ions uptake to seeds because they produced compounds and fine particles and they were strictly incorporated into crystals. Considering metal ion uptake rate per surface area, copper components can take in metal ions rapidly but nickel component cannot so fast. In addition, target metal mole ratio was improved by from 10 to $20 \%$ by the centrifugation in copper carbonate basic-based experiment.

Furthermore, anions impurities like borate or phosphate ions caused the effect of damaging the surface of seeds or producing many fine particles during the reactive crystallization.

Target and impurity ions both were removed with a high rate (over $99 \%$ ) by this 8 -hour reactive crystallization, and this process concluded to be an effective procedure for the metal ions removal from the wastewater containing several kinds of impurities.

\section{Notation}

A: $\quad$ Seed surface area $\left(\mathrm{m}^{2}\right)$

$\mathrm{m}: \quad$ Metal mole in seed ( $\mathrm{mol})$

$M: \quad$ Metal mole ratio in seed (-)

$N$ : $\quad$ Number of seed crystals(\#)

$r$ : $\quad$ Metal ions uptake rate $\left(\mathrm{mol} \mathrm{s}^{-1}\right)$ 


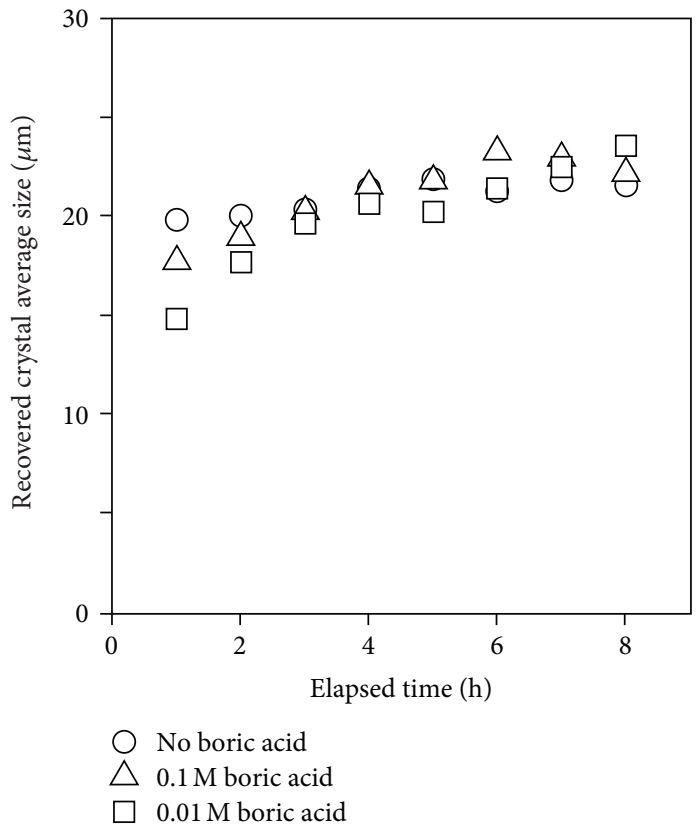

(a)

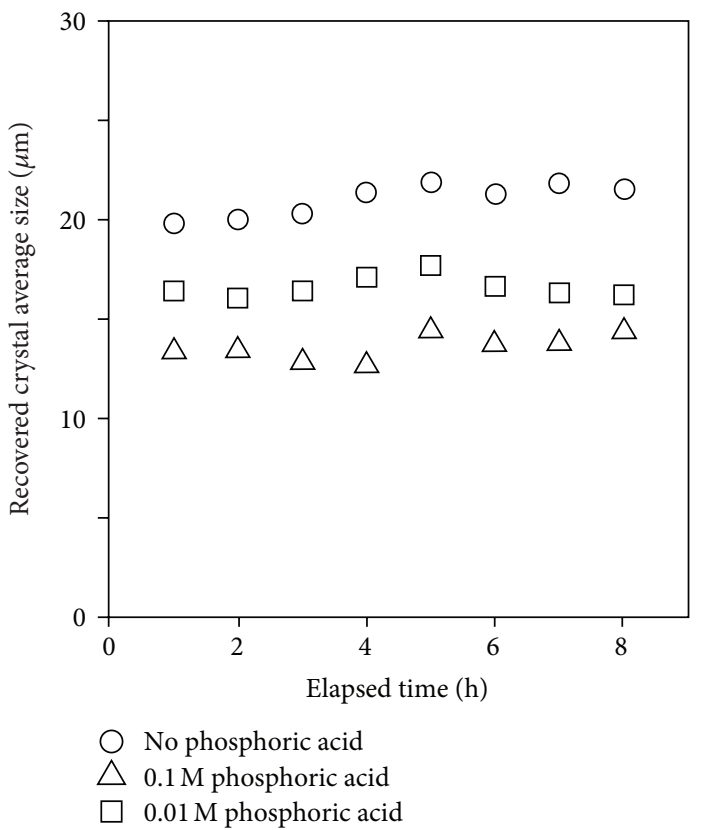

(c)

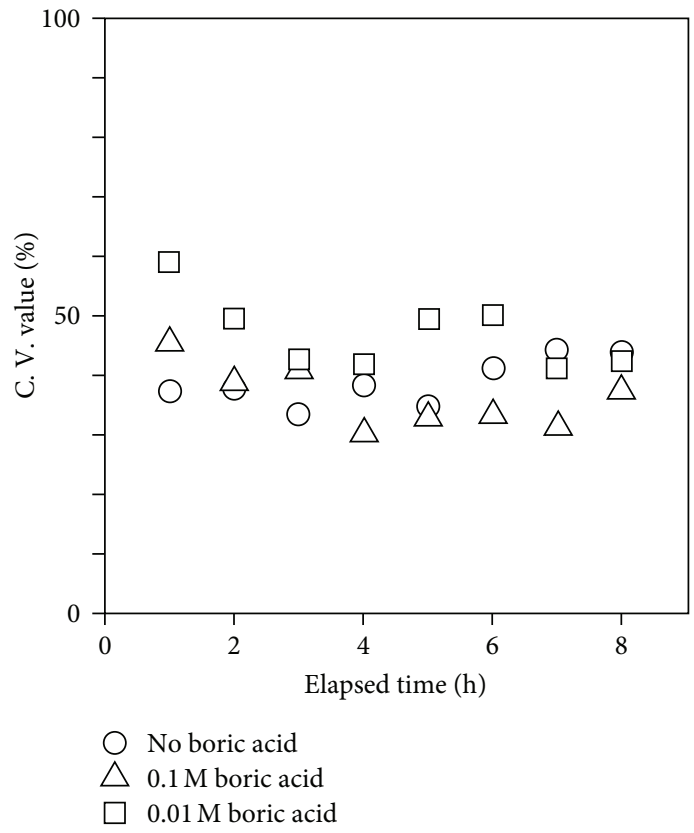

(b)

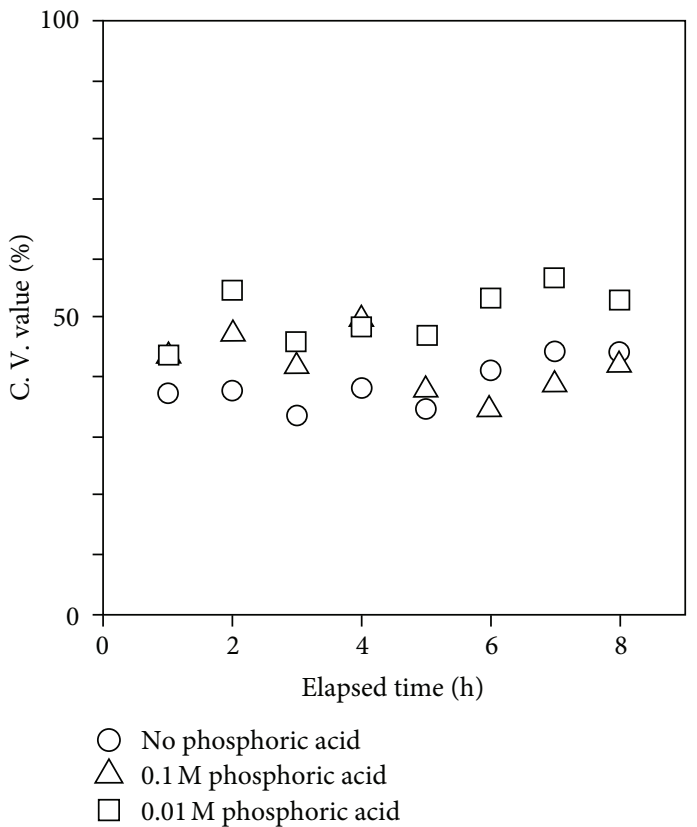

(d)

FIGURE 7: The time variation of produced crystal average size and C. V. value in adding anions as impurities ((a, b) in providing borate ions as impurities, (c, d) in providing phosphate ions as impurities).

$R: \quad$ Metal ions uptake rate per surface area $\left(\mathrm{mol} \mathrm{m} \mathrm{s}^{-1}\right)$

$t$ : Operation time (s).

Index

0: Initial

I: $\quad$ Impurity metal ions

T: $\quad$ Target metal ions

unit: Mono.

\section{Acknowledgment}

This study was established as a result of financially supporting and training in many aspects by the Global COE program "Center for Practical Chemical Wisdom" by MEXT. Also the authors gratefully acknowledge the designing and creating the custom-ordered tank of the Fujimoto Kagaku Co., Ltd. 


\section{References}

[1] J. G. Dean and F. L. Bosqui, "Removing heavy metals from waste water," Environmental Science and Technology, vol. 6, no. 6, pp. 518-522, 1972.

[2] C. I. Lee and W. F. Yang, "Heavy metal removal from aqueous solution in sequential fluidized-bed reactors," Environmental Technology, vol. 26, no. 12, pp. 1345-1353, 2005.

[3] V. C. T. Costodes and A. E. Lewis, "Reactive crystallization of nickel hydroxy-carbonate in fluidized-bed reactor: fines production and column design," Chemical Engineering Science, vol. 61, no. 5, pp. 1377-1385, 2006.

[4] R. P. van Hille, K. A. Peterson, and A. E. Lewis, "Copper sulphide precipitation in a fluidised bed reactor," Chemical Engineering Science, vol. 60, no. 10, pp. 2571-2578, 2005.

[5] Y. Shimizu and I. Hirasawa, "Effect of seeding on metal Ion recovery from wastewater by reactive crystallization of metal carbonates," Chemical Engineering \& Technology, vol. 35, no. 9, pp. 1588-1592, 2012.

[6] I. Uzun and F. Guzel, "Adsorption of some heavy metal ions from aqueous solution by activated carbon and comparison of percent adsorption results of activated carbon with those of some other adsorbents," Turkish Journal of Chemistry, vol. 24, pp. 291-297, 2000.

[7] S. A. El-Korashy, "Studies on divalent ion uptake of transition metal cations by calcite through crystallization and cation exchange process," Journal of Materials Science, vol. 38, no. 8, pp. 1709-1719, 2003.

[8] S. K. Handona and U. A. Al Hadad, "Crystallization of calcium sulfate dihydrate in the presence of some metal ions," Journal of Crystal Growth, vol. 299, no. 1, pp. 146-151, 2007.

[9] K. Soya, N. Mihara, D. Kuchar, M. Kubota, H. Matsuda, and T. Fukuta, "Use of caffeine and human pharmaceutical compounds to identify sewage contamination," International Journal of Civil and Environmental Engineering, vol. 2, no. 2, pp. 93-97, 2010.

[10] T. Fukuta, T. Ito, K. Sawada, Y. Kojima, H. Matsuda, and K. Yagishita, "Improvement of nickel-precipitation from aqueous nickel solution by sulfuration with sodium sulfides," Journal of Chemical Engineering of Japan, vol. 36, no. 4, pp. 493-498, 2003. 

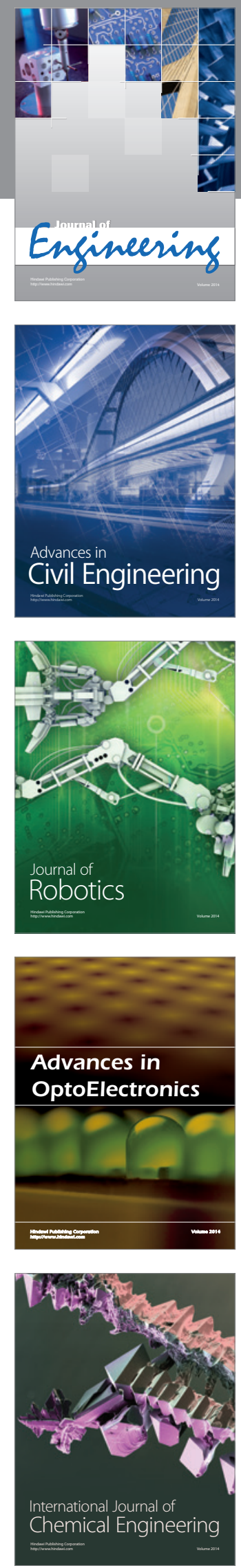

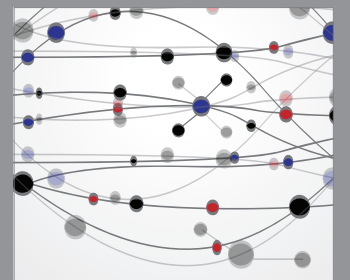

The Scientific World Journal
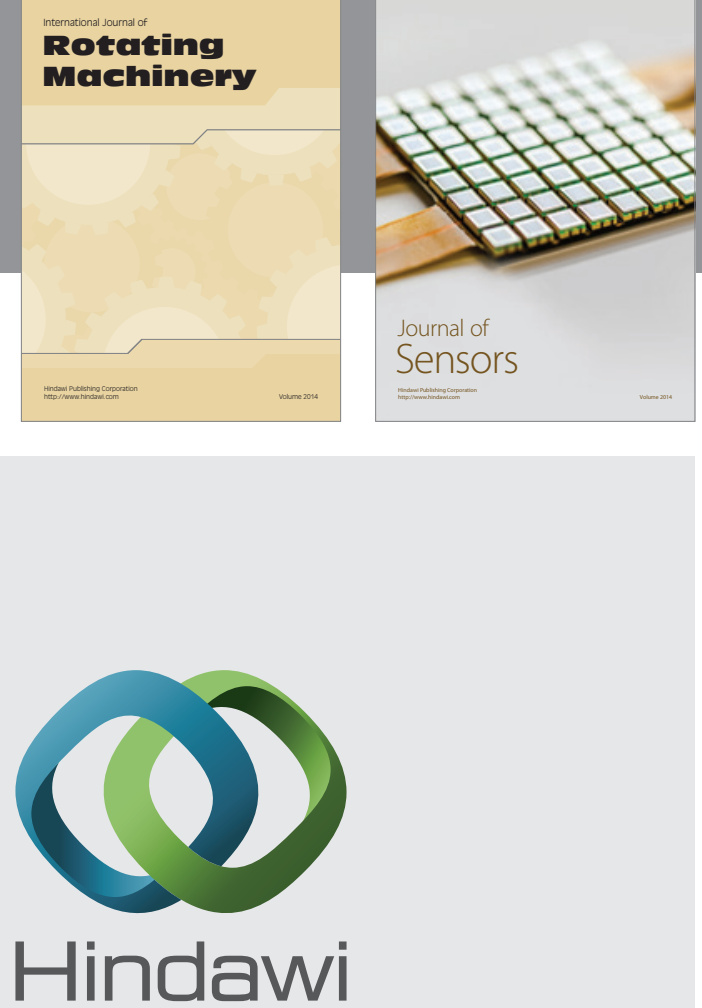

Submit your manuscripts at http://www.hindawi.com
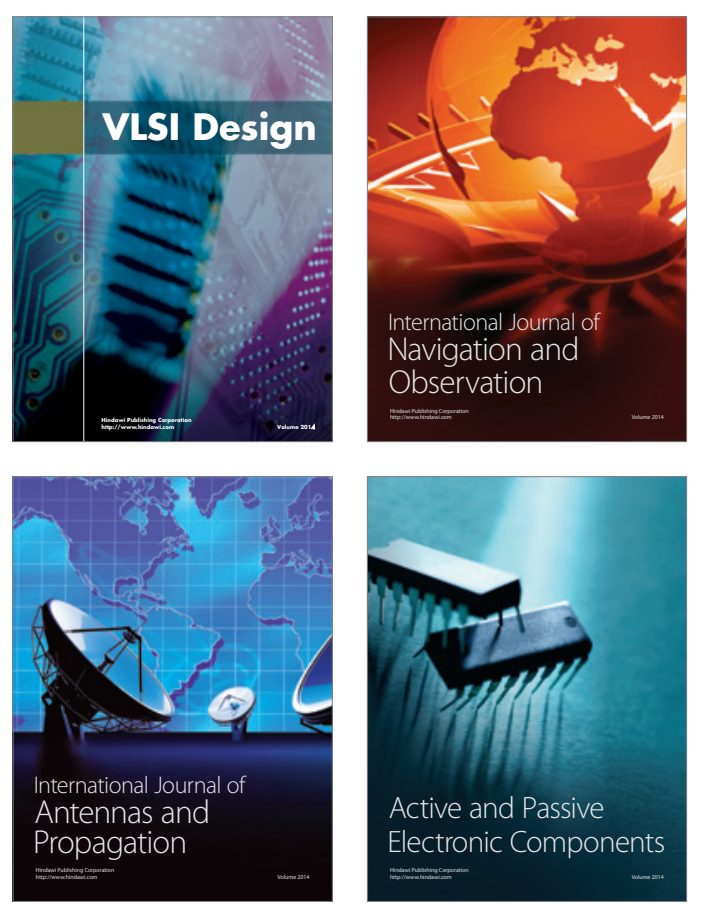
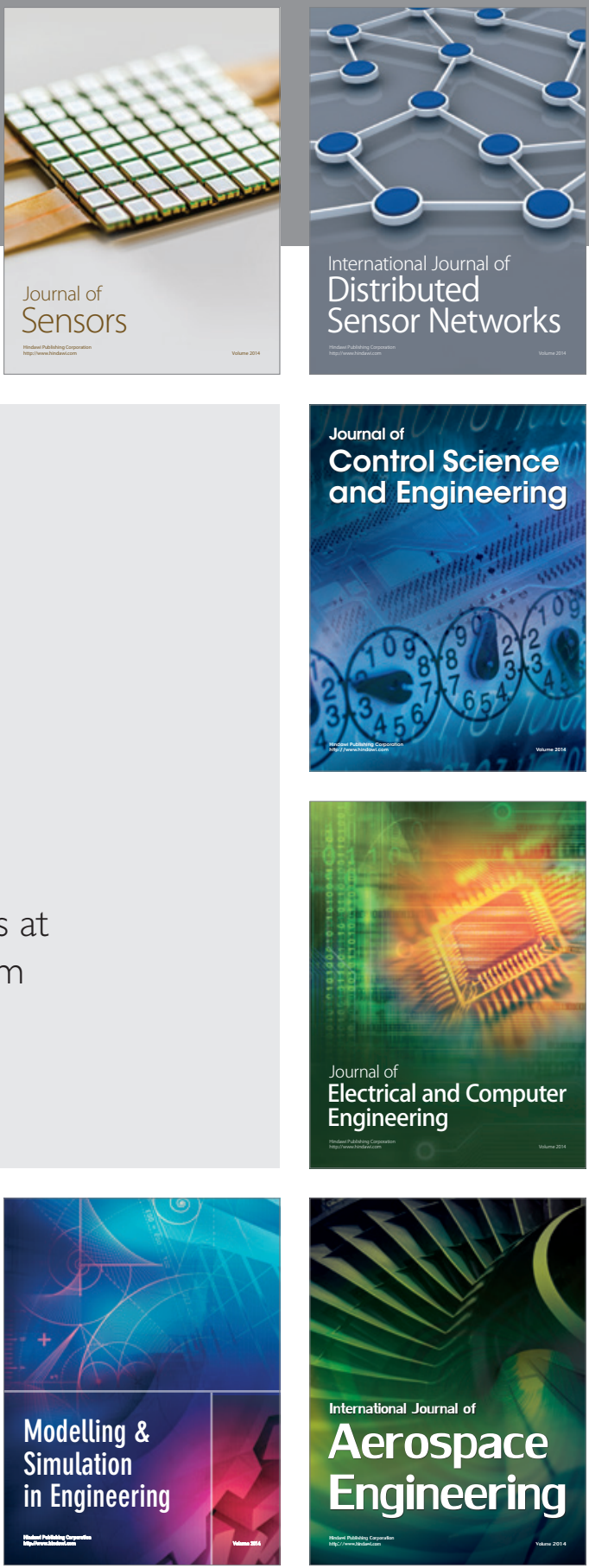

Journal of

Control Science

and Engineering
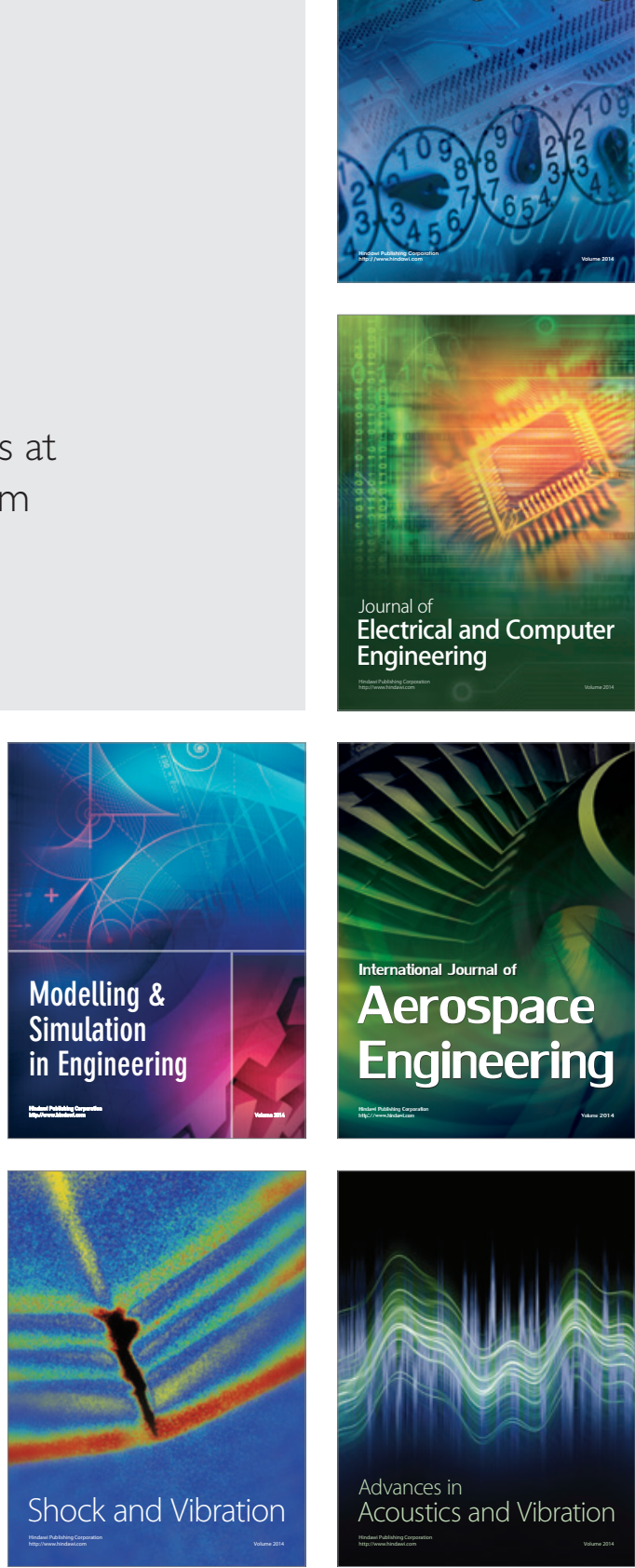